ORIGINAL ARTICLE

AFRICAN JOURNAL OF CLINICAL AND EXPERIMENTAL MICROBIOLOGY AJCEM/201179/21203

COPYRIGHT 2012

AFR. J. CLN. EXPER. MICROBIOL 13(2): 79-83 http://dx.doi.org/10.4314/ajcem.v13i2.4

\title{
EVIDENCE OF LONG TERM BENEFIT OF PRAZIQUANTEL TREATMENT AGAINST SCHISTOSOMA MANSONI IN KIGUNGU FISHING VILLAGE OF ENTEBBE, UGANDA
}

\author{
Running title: Praziquantel treatment against Schistosoma mansoni in Kigungu fishing village of Entebbe, \\ Uganda
}

Emmanuel I Odongo-Aginya ${ }^{1}$; Kironde F.; Lyazi,MI ${ }^{3}$; Kyabayinze, $\mathrm{D}^{4}$; Sempewo, Harman ${ }^{5}$; Male,Alan ${ }^{6}$; Rodrigo Correa Oliveira ; Wilson, Alan ${ }^{8}$

\begin{abstract}
1. Department of Microbiology, Gulu University, P.O.Box 166 Gulu, UGANDA, 2. Biochemistry Department, Makerere University, P.O.Box 7062 Kampala, UGANDA 3. Statistic Child Health Department, Mulago-Makerere, UGANDA., 4. Clinical epidemiology Unit, Faculty of Medicine, Makerere University, P.O.Box 7062. Kampala, UGANDA; 5. Nsambya Missionary Hospital, Kampala, P.O.Box 7146, Kampala UGANDA; 6.National Agricultural Biotechnology Centre, P.O.Box 6247 Kampala, UGANDA; 7. Centro de Rene, Rachou-Fiocruz Laboratorio de Immologia Cellular e Molecular, Av.Agusto de Lima, 1715 Barr Preto, CEP 30190-002 Belo Horizonte, Minas Gerais, Brazil. 8. University of York, Northumberland House, 303-306 High Holborn, London, WC1V 7JZ, United Kingdom.
\end{abstract}

Correspondence: Odongo-Aginya, Emmanuel, Gulu University, P.O.Box 166 Gulu, Uganda (TELEPHONE 256 0772505761. email eodongoaginya@yahoo.com

Abstract.

Praziquantel (PZQ) is efficacious against all species of schistosome: Schistosoma mansoni; Schistosoma haematobium; Schistosoma japonicum and other parasites like the Taenia species. This cross-sectional cohorts study was carried out in Kigungu fishing village along Lake Victoria shore in Entebbe Uganda. Our analysis was based on examining microscopically three slides from a single stool specimen from each of base line cohorts 945 .These included children and adults, participants from both sexes in Kigungu fishing village in Entebbe Uganda. Nine hundred and one (901) of the cohorts were re-examined after six months and 625 of the same cohorts who were examined at the baseline and after six months were re-examined 18 months later. The slides were prepared using modified Kato/Katz (Odongo-Aginya) method. The infection proportion with Schistosoma mansoni at baseline was $448(47.5 \%)$ but this was reduced to $244(25.8 \%) 18$ months after treatment with a single oral dose of praziquantel at $40 \mathrm{mg} / \mathrm{kg}$. However $495(52.5 \%)$ were negative at the baseline study. The cure proportion after six was significant $\{(\mathrm{P}=0.00),(\mathrm{OR} 4.63) \mathrm{CI}$ at $95 \%(3.53-6.06)\}$. Similarly the cure proportion after 18 months was significant $\{(\mathrm{P}=0.00)$, (OR2.2) CI at $95 \%(1.87-3.34)\}$. The force of re-infection after six months was significant $\{(P=0.0001),(O R 0.47) C I$ at $95 \%(0.31-$ $0.71)\}$. Nevertheless the force of re-infection was not significant after 18 months $\{(P=0.766),(O R 0.95) C I$ at $95 \%(0.68-1.34)\}$ eggs excretion did not reach the level of the pre-treatment intensity. The egg reduction was $69.3 \%$. This was associated with age and pre-treatment intensity $<400$ eggs per gram $(\mathrm{epg})$ of faeces and age groups $\geq 30$ years. The egg reduction also resulted in marked decrease in clinical symptoms in the participants. Our study suggests evidence of long-term benefit of praziquantel in Kigungu and that the re-infection occurred more commonly in younger age group than in the older patients.

Key words: Praziquantel; Schistosoma mansoni; Kigungu; Entebbe; Uganda.

\section{Introduction}

Praziquantel (PZQ) is efficacious against Schistosoma mansoni; Schistosoma haematobium; Schistosoma japonicum and other parasites like the Taenia species (1). Presently in Uganda, one of schistosomiasis endemic countries in sub Saharan Africa, PZQ is the drug of choice in controlling morbidity due to schistosomiasis because the mean cost of treatment per dose per person in Uganda is $\$ 0.3(1,2,3)$. In spite of its low cost, Uganda is still unable to procure adequate PZQ for mass chemotherapy (2). Consequently it was found necessary to establish a treatment regimen for revaluation study in Uganda. Previous studies in Uganda and elsewhere revaluated the efficacy of PZQ after six months to establish the cure proportion $(4,5)$. Nevertheless other studies revaluated the efficacy of PZQ at the interval of one year for a longer period $(6,7)$. At present, studies in different endemic settings using the single oral dose regiment of $40(\mathrm{mg} / \mathrm{kg} \mathrm{wt})$ of PZQ against S. mansoni, S. haematobium and mixed infections, the efficacy of
PZQ is reported to stand at $60-90 \%$ (8). Extensive use of PZQ in Uganda and elsewhere in the tropics has been linked to the development of parasite resistance to the drug. This is evidenced by reduced cure proportion in humans treated with PZQ in the Richard Toll area of Senegal (3). However, factors like intensity of infections and high transmission of infections have been known to influence schistosomicidal activity of PZQ(4). Pharmacologically, Praziquantel kills adult worms by penetrating and damaging tegument of the adult worms (9). Subsequently there is a transient increase of antigens released from the dead adult worms and egg antigens in the circulation, which stimulate humoral and cellular immune responses of the host $(10,11)$. These anti-worm and anti-egg responses in the host aid in the killing of the worms by antibody binding to the antigen exposed due to Praziquantel damaged tegument (12). This phenomenon may continue for long after the pharmacological effect of 
PZQ (9). Repeated treatment with PZQ has been shown to improve cure proportion as observed in West Nile districts of Uganda $(13,14)$. These are districts with high transmission and high intensity of infection $(13,15)$.

This study investigated the effect of PZQ on cure proportion and egg reduction 18 months after treatment in Kigungu-fishing village, a community with high exposure risk to $S$. mansoni infection in order to establish cost effective treatment regimen that could be adopted in Uganda schistosomiasis mansoni endemic foci (16).

\section{MATERIALS AND METHODS}

Study sample

A baseline evaluation study was carried out on a cohort of 945 volunteer adults and children who were residents of Kigungu fishing village, Entebbe, Uganda. Enrolled 448 patients were stool positive for S. mansoni while enrolled 495 patients were stool negative for $S$. mansoni. Treatment was administered to the $448 \mathrm{~S}$. mansoni positive patients. Six months later $95.5 \%$ (901/945) patients were reviewed. These consisted of $433(96.7 \%)$ patients from the $448 S$. mansoni positive patients in the base line survey (fifteen were lost). Out of the 433, forty were excreting eggs of $S$. mansoni in their stool. They were treated and left out of the study. Therefore people who remained eligible for the study in this group were 393. Similarly, 468 (94.5\%) among the 495 negative patients at the baseline were reviewed (see flow chart below). Ninety two of these 468 previously negative patients were subsequently (6 months later) also found with eggs of S.mansoni in their stool. They were also treated with $40 \mathrm{mg} / \mathrm{kg}$ body weight and left out of the study. Therefore people who remained eligible for the study in this group were 376 . Of the 393 S. mansoni positive reviewed after six months, 83 people were lost to follow up only 310 patients came for follow-up and 80 patients were stool positive for S. mansoni while patients 230 were still stool negative for S. mansoni. Of the $376 \mathrm{~S}$. mansoni negative after six months (not treated), 61 people were lost to follow up only 315 patients came for follow-up, and 102 Patients had become infected with $S$. mansoni while 213 patients remained stool negative for S. Mansoni throughout the 18 months' follow up. The participants were registered in the three studies using their study code numbers, names, sex, age, home locations and the names of head of the families.

Figure 1: Flow chart showing the follow up of patients from first recruitment up to 18 months

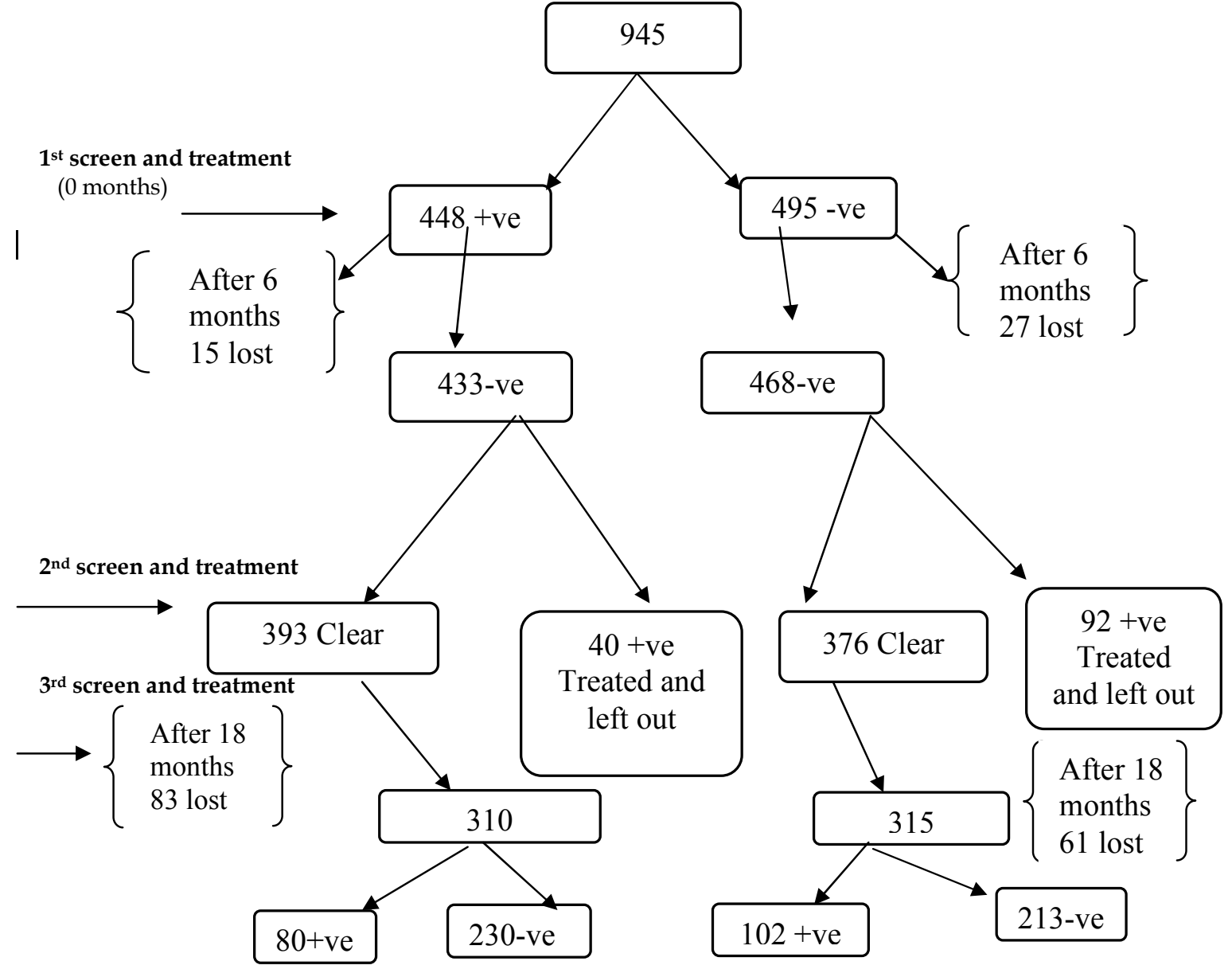




\section{Study area}

This study was conducted in Kigungu fishing village, situated along Lake Victoria in Entebbe peninsula. This village is located to the extreme end of the peninsula, at latitude $35^{0}$ to $38^{\circ}$ East and $03^{0}$ to $07^{0}$ North. It is about 15 kilometers from Entebbe Municipality and about half a kilometer from the Entebbe international airport. This fishing village was selected because it had base line data on prevalence and intensities of $S$. mansoni and other soiltransmitted helminths (16). The population of Kigungu is estimated to be 6,000 people with nearly an equal sex 1:1 ratio. Their main occupation is fishing. Besides fishing, they do a little subsistence farming mostly for food crops. Their water exposure is high and hence is the source of infection and reinfection.

\section{Procedure of the study}

Informed consent was obtained from all the participants. Residents who had consented to participate in the study and children between 5 and 18 years old who had been granted permission to participate in the study by their parents/guardians and have not taken antischistosomal treatment six months prior to the base line study were registered. This explains the 55 people who were excluded from the base line registration. Those giving their consent and were literate, were asked to sign an official form showing acceptance. Meanwhile the illiterate patients used thumb prints on official form showing acceptance. All participants who consented to the study and were found $S$. mansoni positive were administered free antischistosomal therapy and treatment for any other ailments. Patients unwilling to participate in the study were not penalised in any way and normal clinical services and treatments including antischistosomal therapy were not conditioned to the patient's participation in the proposed research work. Medical doctors, and nurses, carried out health examinations and recorded the various clinical symptoms. Medical laboratory technologists carried out laboratory investigations and the social worker coordinated the interviews and recruitment. All patients infected with S. mansoni were treated at the study site with praziquantel (Medochemie Ltd.Limassol-Cyprus Europe) at $40 \mathrm{mg}$ per $\mathrm{kg}$ body weight. Illnesses, other than schistosomiasis, detected during examination were appropriately treated or referred to other health facilities. Patient's privacy was duly respected.

Residents for the study reported to the clinic between 9 a.m and 2 p.m of our publicised workdays. They were interviewed and examined by a physician and a nurse during initial screening. The physical examination included special attention to status of the abdominal organs commonly affected by $S$. mansoni worm. Anaemia and fever were also recorded. Patients with body temperature greater than $37.5^{\circ} \mathrm{C}$ had blood smear test for malaria parasites done. On each working day, thirty consecutive patients in the order of their arrival at the clinic were registered into the study.

\section{Determination of intensity of infestation by} intestinal worms

A stool container labelled with individual code number, name, age and sex was given to each patient to return with about 5-10 gram of stool specimen. Eggs in the stool were quantified using modified Kato-Katz method (17). Essentially, each stool specimen was initially strained through a stainless steel sieve of $250 \mu \mathrm{m}$ mesh size to remove artefacts. The stained sample was filled in a template, a device measuring $41.7 \mathrm{mg}$ of stool. Each of three separate aliquots of $41.7 \mathrm{mg}$ stool was sampled on a different glass microscope slide and processed for examination as follow. About $10 \mu \mathrm{l}$ of compound stain consisting of eosin $5 \%$ eosin, and $7.5 \%$ nigrosin in $10 \%$ formalin was added to stool smear on each slide. The stain was stirred in the stool smear on the slide using tooth pick. A wettable cellophane cover slip (32 x 41 $\mathrm{mm}$ ) pre-soaked in 50\% glycerine was carefully placed on the stained stool smear and gently pressed down. The excess stain from the smear on slide was blotted out with absorbent paper before the prepared slide was read using objective $\times 10(17,18)$. The arithmetic mean of the eggs counted in three slides was recorded as the count in 41.7 milligram of stool. To convert the mean egg count into egg per gram of faeces a factor of 24 was multiplied by the mean of the eggs counted \{i.e. number of eggs (n) x $1000 \mathrm{mg} / 41.7$ $\mathrm{mg}=24 \times \mathrm{n}=$ eggs per gram faeces $\}$. Intensity of infection was classified as follows: low $=1-100$ eggs per gram, medium $=101-400$ and high $=\geq 401$ eggs per gram of stool (6).

\section{Data management and statistical analysis}

Data were double entered using Microsoft Excel and crosschecked by different researchers. The arithmetic means egg counts of the individuals were categorised according to infection intensities as follows 0-100; 101200; 201-300; 301-400; and $\geq 401$. Similarly age group was also categorised into five groups 5-10; 11-20; 21 30; $31-40$ and $\geq 41$. The percent of cured proportion was calculated from individuals who had no $S$. mansoni egg in their stool after treatment divide by the total number of individuals who had S. mansoni egg in their stool before treatment multiply by $100 \%$. The percentage of egg count reduction was calculated from the arithmetic mean egg count after and before treatment. Pearson's Chi-X 2 was used to find out associations between a pair of categorical data at $95 \%$ level of confidence while Pearson's and Spearman's rank correlations were used for comparison of continuous data.

\section{RESULTS}

In this study, we recruited nine hundred and forty five residents, adults and children of both sexes. They 
were all from Kigungu village of Entebbe in Uganda. Four hundred and forty eight $(47.5 \%)$ were S. mansoni positive at the baseline survey and they were treated with a single dose of praziquantel at $40 \mathrm{mg} / \mathrm{kg}$ body weight. Four hundred and ninety five $(52.5 \%)$ were negative for S. mansoni. Six months later, the study participants were reviewed for treatment success in clearing the infection. Nine hundred and one (95.5\%) of the people who participated in the baseline survey came back for the review but $4.5 \%$ (mostly school children relocated themselves elsewhere). A very small proportion $(2.4 \%)$ of the 448 patients who were S. mansoni positive at the baseline survey continued to excrete eggs of $S$. mansoni in their faeces. Similarly $2.5 \%$ of the 468 negative participants at the baseline survey were infected. The $S$. mansoni positive participants were again treated with $40 \mathrm{mg} / \mathrm{kg}$ body weight and were left out of the study. After follow up of participants from the seventh to the eighteenth months, 625 ( 310 from positive +315 from negative) of them came back for the third evaluation while additional 276 participants were lost to follow up.

Table 1, shows the distribution of intensity of infection among 80 male and female participants who were found infected in the baseline study, treated six months later and still got re-infected and thus found to be stool positive at end of 18 months of follow up . At the same time, the force of clearance by PQZ was $230(74.2 \%)$ \{i.e. 230 from 310 \} this was the total number of patients who were negative for S. mansoni after 18 months. Table 2, Compares difference between those who remained negative after treatment with those who were infected in the baseline study the force of clearance of PZQ showed significant difference $(P=0.00)$. Similarly the force of clearing the infection of PZQ after 18 was also significant $(\mathrm{P}=0.00)$. While the re-infection rate was significant $(\mathrm{P}=0.0001)$ after six months there was no significant difference in the re-infection rate after 18 months $(\mathrm{P}=0.766)$.

TABLE 1: INTENSITY OF S. MANSONI AMONGST 80 PATIENTS AT RECRUITMENT AND 18 MONTHS LATER ACROSS AGE GROUP IN YEARS.

\begin{tabular}{||l|l|l|l|l|l|l||}
\hline \hline \multicolumn{2}{||l}{ S. MANSONI AT RECRUITMENT, N=80 } & \multicolumn{2}{l||}{$\begin{array}{l}\text { REINFECTION WITH S. MANSONI 18 MONTHS } \\
\text { LATER, N=80 }\end{array}$} \\
\hline Age group & $\mathrm{N}$ & $\%$ & †(mean) & $\mathrm{N}$ & $\%$ & †(mean) \\
\hline $5-10$ & 14 & 17.50 & $9,592(685.1)$ & 14 & 17.50 & $4,851(346.5)$ \\
\hline $11-20$ & 45 & 56.25 & $20,283(450.7)$ & 45 & 56.25 & $9,853(219.0)$ \\
\hline $21-30$ & 12 & 15.00 & $9,845(820.4)$ & 12 & 15.00 & $2,857(238.0)$ \\
\hline $31-40$ & 7 & 8.75 & $6,129(875.6)$ & 7 & 8.75 & $1447(206.7)$ \\
\hline$\geq 41$ & 2 & 2.50 & $2,096(349.3)$ & 2 & 2.50 & $144(72.0)$ \\
\hline TOTAL & 80 & 100 & $47945(599.3)$ & 80 & 100 & $16197(225.0)$ \\
\hline \hline
\end{tabular}

Legend: $\%=$ Percentage of infections in the age group. $\uparrow$ : Total number of eggs excreted by each age group before and 18 months after the third treatment. The figures in parenthesis represent arithmetic mean of the egg count. $\mathrm{N}$ : the total number individuals infected with $S$. mansoni before and after the third treatment.

\section{TABLE 2: CURE RATES AND REINFECTION RATES}

\begin{tabular}{|c|c|c|c|}
\hline Cure rates after 6 months & & P -value & OR (CI) \\
\hline $393 / 433$ & $92 / 468$ & 0.00 & $4.63(3.53-6.06)$ \\
\hline \multicolumn{4}{|l|}{ Cure rates after 18 months } \\
\hline $210 / 310$ & $102 / 376$ & 0.00 & $2.20(1.87-3.34)$ \\
\hline \multicolumn{4}{|l|}{ Re infection rate after 6 months } \\
\hline $40 / 432$ & $92 / 469$ & 0.0001 & $0.47(0.31-0.71)$ \\
\hline \multicolumn{4}{|l|}{ Re infection rate after 18 months } \\
\hline $80 / 310$ & $102 / 376$ & 0.766 & $0.95(0.68-1.34)$ \\
\hline
\end{tabular}

Legend: cure rate after 6 months and 18 months against force of reinfection in six months and 18 months after treatment 


\section{DISCUSSION}

Several studies of infection and reinfection with Schistosoma mansoni after treatments with PZQ of residents living in endemic areas have shown that after treatment, prevalence and intensity of parasite infestation due to re-infection are lower than before treatment. In other words, the re-infection prevalence and intensity have been shown never to equal before treatment level $(5,7)$. In this cohort study to establish regiments for PZQ treatment against S. mansoni among the residents of Kigungu, showed that there was reduction in the percentage of infection from $47.4 \%$ (448/ $/ 945)$ to $25.8 \%(80 / 310)$ and eggs excretion was reduced by $92.7 \%$. This was 18 months after the initial treatment with PZQ $40 \mathrm{mg} / \mathrm{kg} / \mathrm{wt}$.

We deliberately set a period of 18 months in total with the first six months for the assessment of the success of baseline treatment. We subsequently followed the cohorts twelve months later to allow the study residents exposures to infection. This was to find out the level of resistance and susceptibility developed after treatment. Follow up studies to establish the force of reinfection after treatment with PZQ after short interval is well known, $(5,7,8)$. Schistosomiasis control Programmes notably Uganda and in other Tropical countries are based on the single dose administration of PZQ to large communities. Repeated treatment to re-infected individuals is recommended depending on the degree of transmission in the areas (8). In spite of the low cost of PZQ most counties in Africa are unable to procure adequate PZQ for mass chemotherapy. In Uganda, the mean cost of treatment per dose per person is $\$ 0.3$ $(2,19)$. Study of this kind helps to establish treatment regiments for communities in schistosomiasis endemic areas in the tropical countries with meagre budget for helminth control programmes. Mutapi in their study of changes in specific anti-egg antibody levels with PZQ followed their patients after 9 months (20). In addition, Correa-Oliveira in their natural versus drug induced resistance in S. mansoni infections study followed their patients for five years at an interval of one year (7). Repeated treatment with PZQ within short intervals was also found to have no effect on the re-infection period in children in their first decade of life (7). Nevertheless, the cure proportion of PZQ is greater if the treatment is repeated within a short period but the cost of the treatment remains relatively high $(6,10)$. In this study, in spite of a long period of 18 months after treatment, we were still able to detect a cure proportion of $67.7 \%$ (210 out of 310 ). This observed cure proportion, compare well with others of $60-90 \%$ frequently accepted in endemic areas in the tropic using the same PZQ regimen $(12,21)$. On the other hand lower cure proportion of $18-39 \%$ was observed in very intense focus of $S$. mansoni transmission in northern Senegal $(4,22)$. A set up of this kind may need shorter intervals for re-intervention (7). Pre treatment infections categorised according to the levels of intensity showed that 151 patients in Kigungu were in low intensity levels (1-100 epg), 99 of them were in the middle levels of intensity (101-400epg) and only 60 of them excreted high egg count greater than 401 epg. The observation that 213 patients remained uninfected in all studies points out to an interesting situation in which some of the residents live all their lives in S. mansoni endemic areas but do not get the infection. This group of patients commonly known as endemic normal (putative resistance) was always stool negative for S. mansoni eggs (23). The putative resistant people of Kigungu have been found to be stool negative for $S$. mansoni before and after treatment $(23,24)$. Concurrent infection with other intestinal helminths has been shown to affect cure of Praziquantel against schistosome (25). We have observed mixed infection of S. mansoni and other intestinal parasites like hookworm (species identification was not done), Ascaris lumbricoides, and Trichuris trichiura. Our stool analysis was based on a single stool specimen from each patient. In most community-based studies, cure proportion have been estimated based on only one or two slides Kato/Katz reading usually derived from a single stool sample (26). The multiple stool samples procedure is particularly relevant when the overall geometric mean egg count is likely to be low, because it increases the chances of estimating true cure proportion (21).

An unsuccessful effort to eradicate schistosomiasis in the Tropical countries using integrated control methods by World Health Organisation (WHO), lead to the development of morbidity control strategy using antiparasite drugs. However the regiments for treatment for re-infections in different endemic settings remain unclear. In this context we followed our cohorts for 18 months intervening chemotherapeutically using PZQ at base line, after six and 18 months. Reduction in the percentage of infection from $47.4 \%$ to $25.8 \%$ and reduction of eggs excretion by $69.3 \%$ after 18 months raises hope about the long term benefit of praziquantel after treatment in Uganda for large-scale treatment of schistosomiasis.

\section{ACKNOWLEDGEMENTS}

This consortium study received financial assistant from the European Union grant SCHISTO-M-VAC for which we are indebted. We are grateful to Miss Nalumansi Zaria and Ester Mirembe for handling our patients professionally. We thank all laboratory personnel from Makerere Medical School for their assistance. The invaluable work of the Local councillors Lukwago David and the late Sempala Edward in mobilizing the residents of Kigungu fishing village to participate in the study is highly appreciated. Similarly, we gratefully acknowledge assistance of the Headmaster (Kigungu Primary School) Mr.Sentongo Mustafa and his staff, school pupils and others who registered voluntarily in this study. 


\section{REFERENCES:}

1. Mott, KE., (1982): Control of schistosomiasis morbidity reduction and chemotherapy. Act. Trop., 49, 101-111.

2. Doenhoff, MJ., Kusel, JR., Coles, GC., Cioli, D., (2002): Resistance of Schistosoma mansoni to praziquantel. Is there problem? Trans. Roy. Soc. Trop. Med.Hyg., 96, 465469.

3. Stelma, F., Tall, I., Sow, S., Kongs, A., Niang, M et al., (1995): Efficacy and side effect of praziquantel in an epidemic focus of Schistosoma mansoni. Amer. J. Trop. Med. Hyg., 53, 167-170.

4. Dunne, DW., Butterworth, AE., Fulford, AJC., et al. (1992): Immunity after treatment of human schistosomiasis: association between IgE antibodies to adult worm antigens and resistance to reinfection. Eur. J. Imm., 22, 1483-1494.

5. Odongo-Aginya, EI.,Mugisha,C., (1987): The prevalence of Schistosoma mansoni in migrants from endemic areas living in the peninsula of Entebbe. E. Afr. Med. J., 64 57157.

6. Correa-Oliveira R.,Caldas,IR., et al., (2000): Natural versus Drug-induced Resistance in Schistosoma mansoni infection. Par. Today, 16, 397-399.

7. Mutapi, F., Mduluza, T., et al. (1998): The effect of treatment on the age-antibody relationship in children infected with Schistosoma haematobium and Schistosoma mansoni. Mem. Inst.Oswaldo. Cruz, Rio de Jan., 197 suppl 1,173-180.

8. Davis, A. (1993): Antischistosome drug and clinical practice in Human schistosomiasis. Wallingford $C A B$ International, , editors: 367-404.

9. Cioli, D., Pica Mattoccia, L., (2000): Praziquantel. Parasitol. Res., 90(supp.1), 39.

10. Gryseels, B., (1994): Human resistance to Schistosoma infection: age or experience. Parasitol. Today, $10,380-384$.

11. Utzinger, J., N Goran, EK., N. Dri, A., et al., (2000a): Efficacy of praziquantel against Schistosoma mansoni with particular consideration for intensity of infection. Trop. Med. Intern. Hlth., 5, 771-778.

12. Piquet, Vercruysse, J., Shaw, D.J., Diop, M., et al. (1998): Efficacy of praziquantel against Schistosoma mansoni in northern Senegal. Tran. Roy. Soc. Trop. Med. Hyg., 92, 9-93.

13. Nelson, G.S (1958a): Schistosoma mansoni infection in the West Nile District of Uganda. Part 1.The incidence of $S$. mansoni infection. E. Afr. Med.l J.. 35, 312319

14. Schwert, J., (1951a): On vesical Bilharzia in the Lango, district (Uganda).
Communication. Tran. Roy. Soc. Trop. Med. Hyg., 44, 501-514.

15. Gazzinelli G. Iramaya, RCV., Lillian, MG., et al. (1992): Immunological Profiles of Patients from endemic areas infected with Schistosoma mansoni. Mem. Instit. Oswaldo Cruz, 87 suppl. IV, 139-142.

16. Odongo-Aginya, EI., Taylor, MG, and Ackers, JP. et al. (1997): A field evaluation of Aginya stain in the Kato Technique for quantification of Helminth eggs in the faeces. Trop. Med. Parasito., 46, 275-277.

17. Mahdi, R., Montreso, A., Ali, F., et al. (1999): Independent evaluation of the Nigrosin-Eosin of the Kato-Katz technique. Trop. Med. Internat. Hlth., 4, 46- 49.

18. Sleigh, A.C., Mott, K.E., Hoff, R. et al. (1985): Three years prospective study of evaluation of mansoni's schistosomiasis in North-East Brazil. Lancet, II, 63-66.

19. Naus, CWA., Booth, M., Jones, FM et al. (2003): The relationship between age, sex, egg count and specific antibody responses against Schistosoma mansoni antigens in Uganda fishing community. Tran. Roy. Soc. Trop. Med. Hyg., 8:561-568.

20. Ana, LTR., Maria, MA G., and Rogerio, AP. (1995): Humural Immune Responses in Acute schistosomiasis mansoni: Relation to morbidity. Clin. Infec. Dis., 21, 608-615.

21. Khalife, J., Capron, M., Capron, A., et al. (1986): Immunity in human schistosomiasis regulation of protective immune mechanisms by IgM blocking antibodies. J. exper. Med., 164, 1626-1640.

22. Hagan, P., Blumenthal, UJ., Dunne, D., et al., 1991: Human IgE, IgG4 and resistance to reinfection with Schistosoma mansoni. Nature, 349; 243-245.

23. Andrews, P., (1986): Prazequantel: mechanisms of anti-schistosomal activity. Phamacology Theory, 29,129-156.

24. Gazzinelli G. Katz, N., Nocha, R.S., et al. 1985 Immune responses during human schistosomiasis mansoni VIII. Differential in vitro cellular responsive to adult worm and schistosomular tegumental preparation. Amer. J. Trop. Med. Hyg., 32, 326-333.

25. Fenwick, A., Savioli, L., Engels, D., et al. (2003): Drugs for control of parasitic diseases: status and development in Schistosomiasis .Trends parasitol, , 19: 509515.

26. Kathrin, F., Lorenz, G., Odongo-Aginya, EI., et al. 1999: Evidence of a long term effect of a single dose of praziquantel of Schistosoma mansoni induced hepatosplenic lesions in Northern Uganda. Amer. J. Trop. Med. Hyg., 60(6), 927-931. 